\title{
Automatic Brain and Tumor Segmentation
}

\author{
Nathan Moon ${ }^{1}$, Elizabeth Bullitt ${ }^{2}$, Koen van Leemput ${ }^{4,5}$, and Guido Gerig ${ }^{1,3}$ \\ 1 Dept. of Computer Science, \\ 2 Dept. of Surgery, \\ 3 Dept. of Psychiatry, University of North Carolina, Chapel Hill, NC 27599, USA \\ 4 Radiology-ESAT/PSI, University Hospital Gasthuisberg, B-3000 Leuven, Belgium \\ ${ }^{5}$ Department of Radiology, Helsinki University Central Hospital, Helsinki, Finland
}

\begin{abstract}
Combining image segmentation based on statistical classification with a geometric prior has been shown to significantly increase robustness and reproducibility. Using a probabilistic geometric model of sought structures and image registration serves both initialization of probability density functions and definition of spatial constraints. A strong spatial prior, however, prevents segmentation of structures that are not part of the model. In practical applications, we encounter either the presentation of new objects that cannot be modeled with a spatial prior or regional intensity changes of existing structures not explained by the model.

Our driving application is the segmentation of brain tissue and tumors from three-dimensional magnetic resonance imaging (MRI). Our goal is a high-quality segmentation of healthy tissue and a precise delineation of tumor boundaries. We present an extension to an existing expectation maximization (EM) segmentation algorithm that modifies a probabilistic brain atlas with an individual subject's information about tumor location obtained from subtraction of post- and pre-contrast MRI. The new method handles various types of pathology, space-occupying mass tumors and infiltrating changes like edema. Preliminary results on five cases presenting tumor types with very different characteristics demonstrate the potential of the new technique for clinical routine use for planning and monitoring in neurosurgery, radiation oncology, and radiology.
\end{abstract}

\section{Introduction}

The segmentation of medical images, as opposed to natural scenes, has the significant advantage that structural and intensity characteristics are well known up to a natural biological variability or the presence of pathology. Most common is pixel- or voxel-based statistical classification using multiparameter images [12]. These methods do not consider global shape and boundary information. Applied to brain tumor segmentation, classification approaches have met with only limited success [3/4] due to overlapping intensity distributions of healthy tissue, tumor, and surrounding edema. Often, lesions or tumors were considered as outliers of a mixture Gaussian model for the global intensity distribution, [5] 6], assuming that lesion voxels are distinctly different from normal tissue characteristics. Other approaches involve interactive segmentation tools [7], mathematical 
morphology [8], or calculation of texture differences between normal and pathological tissue [9].

A geometric prior can be used by atlas-based segmentation, which regards segmentation as a registration problem in which a fully labeled, template MR volume is registered to an unknown dataset. High-dimensional warping results in a one-to-one correspondence between the template and subject images, resulting in a new, automatic segmentation. These methods require elastic registration of images to account for geometrical distortions produced by pathological processes. Such registration remains challenging and is not yet solved for the general case.

Warfield et al. 1011 combined elastic atlas registration with statistical classification. Elastic registration of a brain atlas helped to mask the brain from surrounding structures. A further step uses "distance from brain boundary" as an additional feature to improve separation of clusters in multi-dimensional feature space. Initialization of probability density functions still requires a supervised selection of training regions. The core idea, namely to augment statistical classification with spatial information to account for the overlap of distributions in intensity feature space, is part of the new method presented in this paper.

Wells et al. 12] introduced expectation maximization (EM) as an iterative method that interleaves classification with bias field correction.

Leemput et al. [13,14] developed automatic segmentation of MR images of normal brains by statistical classification, using an atlas prior for initialization and also for geometric constraints. A most recent extension detects brain lesions as outliers [15] and was successfully applied for detection of multiple sclerosis lesions. Brain tumors, however, can't be simply modeled as intensity outliers due to overlapping intensities with normal tissue and/or significant size.

We propose a fully automatic method for segmenting MR images presenting tumor and edema, both mass-effect and infiltrating structures. This method builds on the previously published work done by [1314]. Additionally, tumor and edema classes are added to the segmentation. The spatial atlas that is used as a prior in the classification is modified to include prior probabilities for tumor and edema. As with the work done by other groups, we focus on a subset of tumors to make the problem tractable. Our method provides a full classification of brain tissue into white matter, grey matter, cerebrospinal fluid (csf), tumor, and edema. Because the method is fully automatic, its reliability is optimal.

\section{Expectation Maximization Segmentation}

An algorithm for fully automatic segmentation of normal brain tissue using an expectation maximization (EM) algorithm has been previously developed by [13 14]. This algorithm interleaves probability density function (pdf) estimation for each tissue class (gray matter, white matter, and csf), classification, and bias field correction using the classic EM approach. The equation for the classification step is

$$
p\left(\Gamma_{i}=j \mid y_{i}, \theta\right)=\frac{p\left(y_{i} \mid \Gamma_{i}=j, \theta_{j}\right) p\left(\Gamma_{i}=j\right)}{\sum_{k} p\left(y_{i} \mid \Gamma_{i}=k, \theta_{k}\right) p\left(\Gamma_{i}=k\right)}
$$




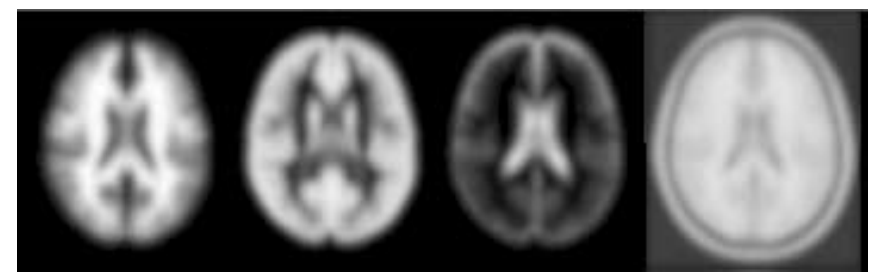

Fig. 1. SPM atlas providing spatial probabilities. From left to right: white matter, gray matter, csf, template T1 image for registration.
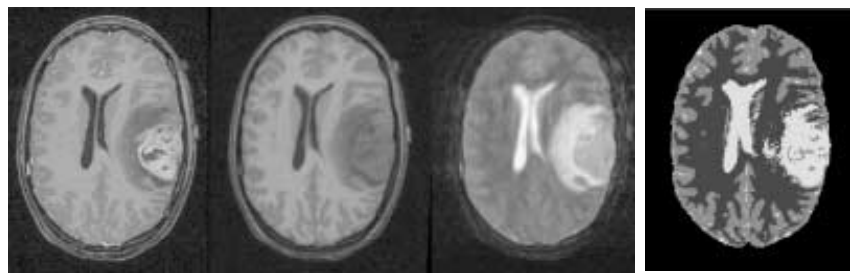

Fig. 2. Left: Registered dataset showing a malignant glioma. From left to right: T1 post-contrast, T1 pre-contrast, T2. The tumor (mostly) enhances with contrast agent in the post-contrast image. Also note the edema surrounding the tumor. Right: Segmentation of dataset using EMS algorithm for healthy brains.

where $y_{i}$ is the intensity at voxel $i$, and $\Gamma_{i}$ is the class of voxel $i$.

The EM segmentation algorithm (EMS) uses a spatial atlas from the Statistical Parametric Mapping (SPM) package for initialization and classification. The SPM atlas contains spatial probability information for brain tissues. It was created by averaging segmentations of normal subjects that had been registered by an affine transformation (Fig. 1).

This spatial atlas is registered to the patient data, with an affine transformation, providing spatial prior probabilities for the tissue classes. The pdfs are then initialized based on the atlas probabilities. This allows the algorithm to be fully automatic. The atlas is also used as the prior probability $p\left(\Gamma_{i}=j\right)$ during the classification step (Eq. 11).

\section{Application of EMS to Tumors}

When a dataset contains a tumor (see Fig. 2 left), there are several problems with the EMS algorithm described in section 2, First, the atlas used does not contain a spatial prior for tumor tissue. The atlas is a normal brain atlas, and cannot be used directly in the presence of pathology. When the atlas is used as a spatial prior for tissue classification, all brain tissue must be classified as one of the available tissue types, either white matter, gray matter, or csf (see Fig. 2 right). Second, tumors are often accompanied by edema, which is a swelling of normal tissue surrounding the tumor, and which changes the tissue properties in that area. The amount and regional extent of edema that accompanies a tumor is variable. Edema or infiltrating phenomena in general are also not explained by the SPM atlas. 
As one would expect, making certain assumptions and using prior knowledge can help greatly in the problem of segmenting brain tumors. We make some important simplifying assumptions for our segmentation framework.

Tumor Characteristics: We assume that tumors are ring-enhancing or fully enhancing with contrast agent. The major tumor classes that fall in this category are meningiomas and malignant gliomas. The basic characteristics of meningiomas are a) smooth boundaries b) normally space occupying and c) smoothly and fully enhancing with contrast agent. The basic characteristics of malignant gliomas are a) ragged boundaries, b) initially only in white matter, possibly later spreading outside white matter, c) only margins enhance with contrast agent, and d) accompanied by edema.

MR Sequences: We assume that all datasets analyzed include a T1 precontrast image, a $\mathrm{T} 1$ post-contrast image (both with $1 \times 1 \times 1.5 \mathrm{~mm}^{3}$ voxel dimensions), and a T2 image $\left(1 \times 1 \times 3 \mathrm{~mm}^{3}\right.$ voxel dimensions) (Fig. 2). This inter-slice spacing is the standard protocol at the hospitals where our datasets were acquired. All of our data are acquired on Siemens 1.5T and 3T scanners.

\section{Extension of the EMS Algorithm}

We have extended the EMS algorithm in three significant ways. The first modification is the addition of a tumor class and an edema class. Second, while the original EMS algorithm is not dependent on the number or type of input channels, our algorithm depends on T1 pre- and post-contrast images. The T1 images are necessary because information is extracted from their difference image. The T2 image simply makes the segmentation more robust. The gadolinium enhanced $\mathrm{T} 1$ image is not used as a channel for classification but only provides information through the difference image. This strategy is reasonable because, in theory, the gadolinium enhanced T1 image does not provide any extra information in addition to the pre-contrast $\mathrm{T} 1$ image except what the difference image provides. Other modalities can be used in addition to the T1 and T2 images. Finally, we modify the spatial atlas to allow for the tumor and edema classes.

Tumor Class: In addition to the three tissue classes assumed in the EMS segmentation (white matter, grey matter, csf), we add a new class for tumor tissue. Whereas the (spatial) prior probabilities for the normal tissue classes are defined by the atlas, the spatial tumor prior is calculated from the T1 pre- and post-contrast difference image. We assume that the (multiplicative) bias field is the same in both the pre- and post-contrast images. Using the log transform of the $\mathrm{T} 1$ pre- and post-contrast image intensities then gives a bias-free difference image, since the bias fields (now additive) in the two images cancel out.

Difference Image Histogram: The histogram of the difference image shows a peak around 0 , corresponding to noise and subtle misregistration, and a positive response corresponding to contrast enhancement (see Fig. 3] left). We would like to determine a weighting function, essentially a soft threshold, that corresponds to our belief that a voxel is contrast enhanced. We calculate a mixture model fit to the histogram. Two Gaussian distributions are used to model the normal 

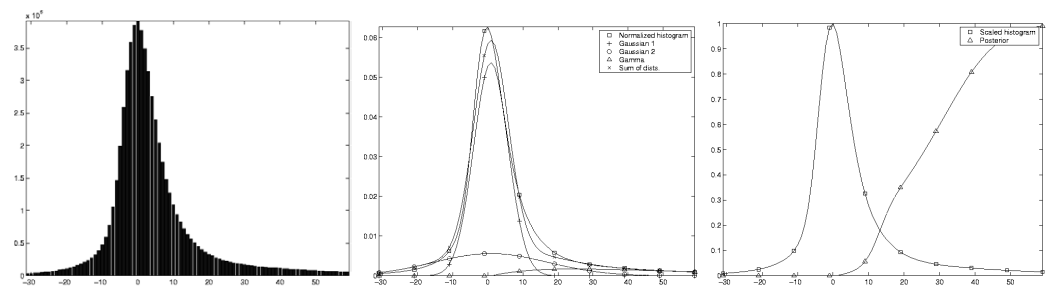

Fig. 3. Left: A T1 pre- and post-contrast difference histogram. Center: The histogram is fit with two Gaussian distributions and a gamma distribution. The gamma distribution represents the enhanced voxels in the post-contrast image. Right: The posterior probability of the gamma distribution (shown along with the scaled histogram) gives the probability that a voxel in the post-contrast image is enhanced. This probability is used to create a spatial prior for tumor tissue.

difference image noise, and a gamma distribution is used to model the enhanced tissue. The means of the Gaussian distributions and the location parameter of the gamma distribution are constrained to be equal (see Fig. 3 center).

Tumor Class Spatial Prior: The posterior probability of the gamma distribution representing contrast enhancement (Fig. 3 right) is used to map the difference image to a spatial prior probability image for tumor. This choice of spatial prior for tumor causes tissue that enhances with contrast to be included in the tumor class, and prevents enhancing tissue from cluttering the normal tissue classes. We also maintain a low base probability $(5 \%)$ for the tumor class across the whole brain region. In many of the cases we have examined, the tumor voxel intensities are fairly well separated from normal tissue in the $\mathrm{T} 1$ pre-contrast and T2 channels. Even when contrast agent only causes partial enhancement in the post-contrast image, the tumor voxels often have similar intensity values in the other two images (see Fig. 2 left). Including a small base probability for the tumor class allows non-enhancing tumor to still be classified as tumor, as long as it is similar to enhancing tumor in the T1 and T2 channels. The normal tissue priors are scaled appropriately to allow for this new tumor prior, so that the probabilities still sum to 1.

Edema Class: We also add a separate class for edema. Unlike tumor structures, there is no spatial prior for the edema. As a consequence, the probability density function for edema cannot be initialized automatically. We approach this problem as follows: First, we have found that edema, when present, is most evident in white matter. Also, we noticed from tests with supervised classification that the edema probability density appears to be roughly between csf and white matter in the T1/T2 intensity space (see Fig. 4). We create an edema class prior that is a fraction of the white matter spatial prior $(20 \%$, obtained experimentally). The other atlas priors are scaled to allow for the edema prior, just as for the tumor prior. The edema and the white matter classes share the same region spatially, but are a bimodal probability density composed of white matter and edema.

During initialization of the class parameters in a subject image, we calculate the estimates for gray matter, white matter, csf, tumor and edema using the 

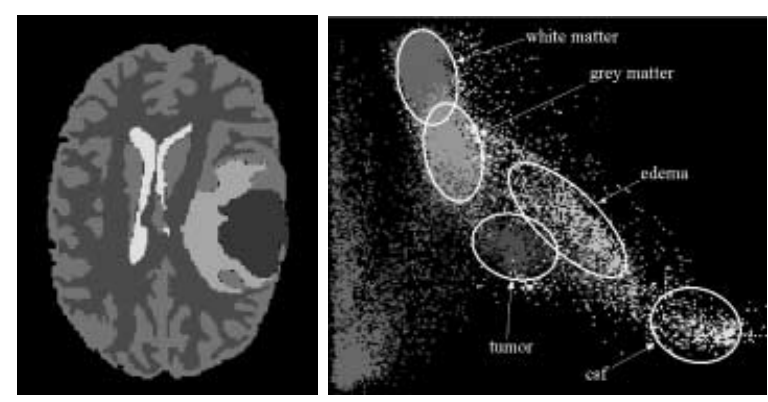

Fig. 4. Feature-space analysis of the T1 and T2 slices from Fig. 2 Left: Segmentation. Right: Scatterplot of the two slices, with colors corresponding to the segmentation. X axis is T2 intensity, $\mathrm{Y}$ axis is $\mathrm{T} 1$ intensity.

modified atlas prior. Thus, white matter and edema would result in similar probability density functions. The bimodal distribution is then initialized by modifying the mean value for edema to be between white matter and csf, using prior knowledge about properties of edema.
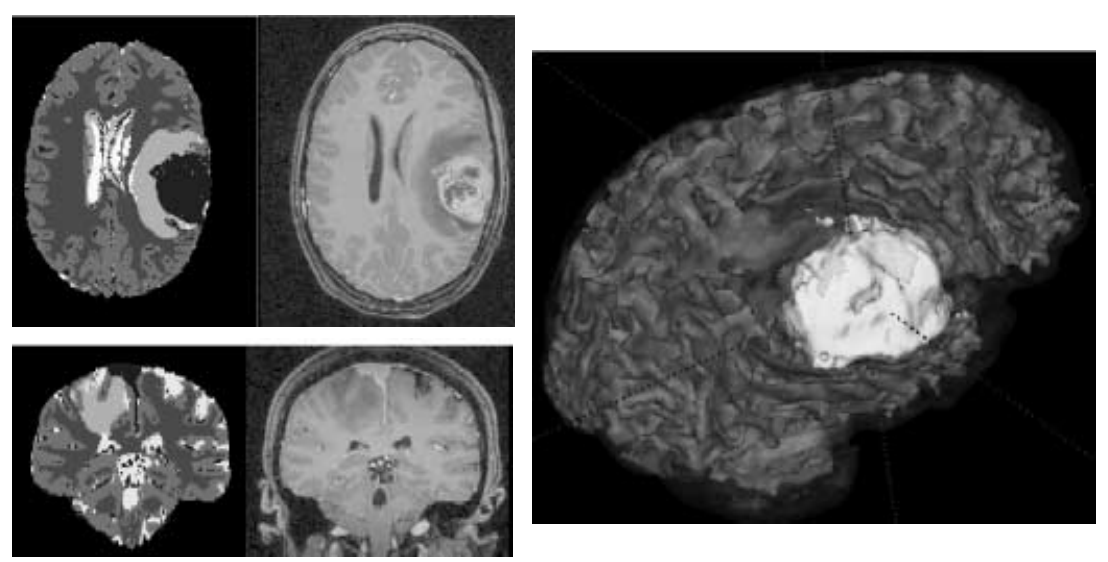

Fig. 5. Left: Two segmented datasets containing malignant glioma (top) and meningioma (bottom), with $\mathrm{T} 1$ post-contrast images for reference. Blue $=$ white matter, green $=$ grey matter, yellow $=$ csf, orange $=$ edema, red $=$ tumor. Right: Three-dimensional rendering of segmented tumor (yellow), white matter tissue (red) and surrounding cortical gray matter (light gray).

\section{Results}

We have applied our tumor segmentation framework to five different datasets, including a wide range of tumor types and sizes. All datasets were registered to the SPM atlas using mutual information registration as described by [16]. Fig. [5] shows results for two datasets. Because the tumor class has a strong spatial prior, many small structures, mainly blood vessels, are classified as tumor because they enhance with contrast. Post-processing is necessary to get a final 


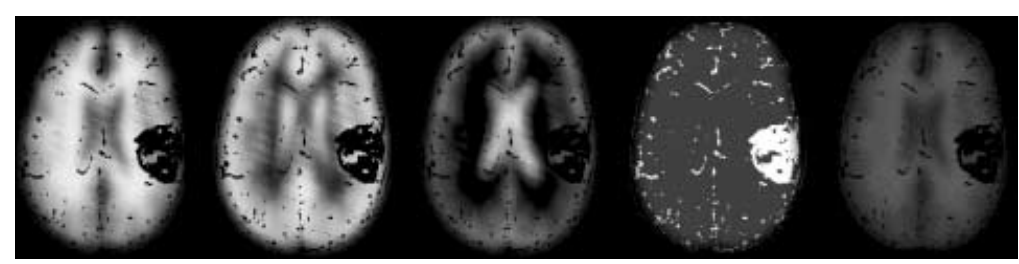

Fig. 6. Spatial prior for the dataset in Fig. 2] created from the SPM atlas and the T1 pre- and post-contrast difference image. From left to right: white matter, grey matter, csf, tumor, edema.

segmentation for the tumor, based on the assumption of large blobs (e.g. using level set evolution [17]). Also note that a small rim around the ventricles is classified as edema, due to partial voluming. This effect would be reduced with higher resolution images. Fig. 6] shows the final spatial priors used for classification of the dataset in Fig. 2 with the additional tumor and edema channels.

\section{Conclusions}

We have developed a model-based segmentation method for segmenting head MR image datasets with tumors and infiltrating edema. This is achieved by extending the spatial prior of a statistical normal human brain atlas with individual information derived from the patient's dataset. Thus, we combine the statistical geometric prior with image-specific information for both geometry of newly appearing objects, and probability density functions for healthy tissue and pathology. Applications to five tumor patients with variable tumor appearance demonstrated that the procedure can handle large variation of tumor size, interior texture, and locality. The method provides a good quality of healthy tissue structures and of the pathology, a requirement for surgical planning or image-guided surgery (see Fig. [5] right). Thus, it goes beyond previous work that focuses on tumor segmentation only.

Currently, we are testing the validity of the segmentation system in a validation study that compares resulting tumor structures with repeated manual experts' segmentations, both within and between multiple experts. A preliminary machine versus human rater validation showed an average overlap ratio of $>90 \%$ and an average MAD (mean average surface distance) of $0.8 \mathrm{~mm}$, which is smaller than the original voxel resolution.

In our future work, we will study the issue of deformation of normal anatomy in the presence of space-occupying tumors. Within the range of tumors studied so far, the soft boundaries of the statistical atlas could handle spatial deformation. However, we will develop a scheme for high dimensional warping of multichannel probability data to get an improved match between atlas and patient images.

\section{Acknowledgments}

This work was supported by NIH-NCI R01CA67812. We acknowledge KU Leuven for providing the MIRIT image registration package. 


\section{References}

1. Just, M., Thelen, M.: Tissue characterization with T1, T2, and proton density values: Results in 160 patients with brain tumors. Radiology (1988) 779-785

2. Gerig, G., Martin, J., Kikinis, R., Kubler, O., Shenton, M., Jolesz, F.: Automating segmentation of dual-echo MR head data. In: IPMI. Volume 511. (1991) 175-185

3. Velthuizen, R., Clarke, L., Phuphianich, S., Hall, L., Bensaid, A., Arrington, J., Greenberg, H., Siblinger, M.: Unsupervised measurement of brain tumor volume on MR images. JMRI (1995) 594-605

4. Vinitski, S., Gonzales, C., Mohamed, F., Iwanaga, T., Knobler, R., Khalili, K., Mack, J.: Improved intracranial lesion characterization by tissue segmentation based on a 3D feature map. Mag Re Med (1997) 457-469

5. Kamber, M., Shingal, R., Collins, D., Francis, D., Evans, A.: Model-based, 3$\mathrm{D}$ segmentation of multiple sclerosis lesions in magnetic resonance brain images. IEEE-TMI (1995) 442-453

6. Zijdenbos, A., Forghani, R., Evans, A.: Automatic quantification of MS lesions in 3d MRI brain data sets: Validation of INSECT. In: MICCAI. Volume 1496 of LNCS., Springer (1998) 439-448

7. Vehkomaki, T., Gerig, G., Szekely, G.: A user-guided tool for efficient segmentation of medical image data. In: CVRMED. Volume 1205 of LNCS. (1997) 685-694

8. Gibbs, P., Buckley, D., Blackband, S., Horsman, A.: Tumour volume determination from MR images by morphological segmentation. Phys Med Biol (1996) 2437-2446

9. Kjaer, L., Ring, P., Thomson, C., Henriksen, O.: Texture analysis in quantitative MR imaging: Tissue characterization of normal brain and intracranial tumors at 1.5 T. Acta Radiologic (1995)

10. Warfield, S., Dengler, J., Zaers, J., Guttman, C., Wells, W., Ettinger, G., Hiller, J., Kikinis, R.: Automatic identification of gray matter structures from MRI to improve the segmentation of white matter lesions. Journal of Image Guided Surgery 1 (1995) 326-338

11. Warfield, S., Kaus, M., Jolesz, F., Kikinis, R.: Adaptive template moderated spatially varying statistical classification. In: MICCAI. Volume 1496 of LNCS., Springer (1998) 431-438

12. Wells, W.M., Kikinis, R., Grimson, W.E.L., Jolesz, F.: Adaptive segmentation of MRI data. IEEE TMI 18 (1996) 429-442

13. van Leemput, K., Maes, F., Vandermeulen, D., Suetens, P.: Automated modelbased tissue classification of MR images of the brain. IEEE TMI 18 (1999) 897908

14. van Leemput, K., Maes, F., Vandermeulen, D., Suetens, P.: Automated modelbased bias field correction of MR images of the brain. IEEE TMI 18 (1999) 885-896

15. van Leemput, K., Maes, F., Vandermeulen, D., Colchester, A., Suetens, P.: Automated segmentation of multiple sclerosis lesions by model outlier detection. IEEE TMI 20 (2001) 677-688

16. Maes, F., Collignon, A., Vandermeulen, D., Marchal, G., Seutens, P.: Multimodality image registration by maximization of mutual information. IEEE-TMI (1997) 187-198

17. Ho, S., Bullitt, E., Gerig, G.: Level set evolution with region competition: Automatic 3-D segmentation of brain tumors. to appear in Proc. ICPR 2002 (2002) 